\title{
EXTRAPOLATION OF MEAN-FIELD MODELS TO SUPERHEAVY NUCLEI
}

\author{
MICHAEL BENDER \\ Gesellschaft für Schwerionenforschung, Darmstadt, Germany
}

\begin{abstract}
The extrapolation of self-consistent nuclear mean-field models to the region of superheavy elements is discussed with emphasis on the extrapolating power of the models. The predictions of modern mean-field models are confronted with recent experimental data. It is shown that a final conclusion about the location of the expected island of spherical doubly-magic superheavy nuclei cannot be drawn on the basis of the available data.
\end{abstract}

\section{Introduction}

The last decade brought with the synthesis of superheavy nuclei with $Z=110-112$ at GSI (Darmstadt) and JINR (Dubna) a renewal of the interest in the properties of superheavy nucleit.2. These are by definition those nuclei with $Z>100$ which have a negligible liquid-drop fission barrier and are stabilized by quantal shell effects only. The ultimate goal is to reach an expected "island of stability" located around the next spherical doubly-magic nucleus which was predicted to be ${ }_{184}^{298} 114$ thirty years ago $\mathrm{s}^{-1}$. Recent experiments performed at JINR Dubnal give evidence for the synthesis of the neutron-rich nuclides ${ }^{283} 112,{ }^{287-289} 114,{ }^{292} 116$, while at Berkeley three $\alpha$-decay chains attributed to the even heavier ${ }^{293} 118$ were observed 7 . The measured $\alpha$-decay hains of these new nuclides turn out to be consistent with theoretical predictions 1.90 . While earlier superheavy nuclei could be unambiguously identified by their $\alpha$-decay chains leading to already known nuclei, the decay chains of the new-found superheavy nuclei cannot be linked to any known nuclides. The new discoveries still have to be viewed carefully, see e.g. the critical discussion in 11 .

The recent experimental developments are accompanied by a significant progress in the theoretical modeling of superheavy nuclei by means of nuclear mean-field (MF) models. MF models can be divided into two major classes, (i) self-consistent (SC) ones where the single-particle wave functions are calculated from an average nuclear potential which in turn depends on these wave functions and (ii) macroscopic-microscopic (MM) models which are composed by a generalized liquiddrop model that governs the bulk properties and a single-particle potential from which the shell correction is derived.

Recent theoretical work reveals systematic differences among the predictions of the models. While modern refined MM models confirm the older prediction of ${ }_{184}^{298} 114$ for the next spherical doubly-magic nucleus, nearly all SC models shift that property to higher charge numbers, depending on the class of SC models to either

${ }_{172}^{292} 120$ or ${ }_{184}^{310} 126$. The reasons for these conflicting predictions and their implications are the topic of this contribution.

Invited talk at the International Conference on "Fusion dynamics at the extremes", Dubna, Russia, May 25-27, 2000. 


\section{Models}

Most SC mean-field models can be viewed as energy density theories in the spirit of the Hohenberg-Kohn-Sham (HKS) approach 1213 originally introduced for manyelectron systems which is nowadays a standard tool successfully applied in atomic molecular, cluster, and solid-state physics. Starting point is the existence theorem 14 for a unique energy functional $\mathcal{E}$ depending on all local densities and currents that can be constructed from the general single-particle density matrix

$$
\hat{\rho} \equiv \rho\left(\mathbf{r}, \sigma, t ; \mathbf{r}^{\prime}, \sigma^{\prime}, t^{\prime}\right)=\sum_{k} v_{k}^{2} \psi_{k}^{*}\left(\mathbf{r}^{\prime}, \sigma^{\prime}, t^{\prime}\right) \psi_{k}(\mathbf{r}, \sigma, t)
$$

which gives the exact ground-state energy of a system of identical Fermions when $\mathcal{E}$ is calculated for the exact ground-state density $\hat{\rho}$. $\mathbf{r}, \sigma$, and $t$ are the spatial, spin, and isospin coordinates of the wave functions $\psi_{k}$. The HKS approach maps the nuclear many-body problem for the "real" highly-correlated many-body wave function onto a system of independent particles in effective, so-called Kohn-Sham orbitals $\psi_{k}$. The equations of motion of the $\psi_{k}$ are derived from a variational principle

$$
\delta \mathcal{E}=0 \quad \Rightarrow \quad \hat{h}(\mathbf{r}, \sigma, t) \psi_{k}(\mathbf{r}, \sigma, t)=\epsilon_{k} \psi_{k}(\mathbf{r}, \sigma, t)
$$

where the single-particle Hamiltonian $\hat{h}$ is the sum of the kinetic term $\hat{t}$ and the self-consistent potential $\Gamma$ that is calculated from the actual density matrix

$$
\hat{h}=\frac{\delta \mathcal{E}}{\delta \hat{\rho}}=\hat{t}+\hat{\Gamma}[\hat{\rho}]
$$

The existence theorem for the energy functional, however, makes no statement about the actual structure of the effective interaction. Guided by symmetry principles and phenomenological knowledge about nuclei, the aim is to find the most simple energy functional which incorporates all relevant physics and to adjust its parameters uniquely to a selected set of nuclear key data. The two most widely used SC models are the (non-relativistic) Skyrme-Hartree-Fock (SHF) model and the relativistic mean-field (RMF) model. The SHF energy functional contains all bilinear combinations of local densities that are invariant under rotational, translational, parity, and time-reversal trapsformations up to second order in the derivatives plus a simple density dependence 15 . The standard RMF energy functional assumes the nucleus to be a system of Dirac nucleons interacting via scalar and vector fields usmally associated with $\sigma, \omega$, and $\rho$ mesons, again plus a simple density dependence 16 . Pairing is treated in both SHF and RMF using the same (non-relativistic) local pairing energy functional corresponding to a delta pairing force 17 .

MM models can be mptivated as an approximation to SC models by means of the Strutinsky theorem 18 19. The binding energy is separated into a large average part $\tilde{E}$ depending smoothly on $N$ and $Z$ and a small shell correction $E_{\text {shell }}$ that describes local fluctuations of the binding energy caused by variations of the density of single-particle levels around the Fermi surface

$$
E(Z, N)=\tilde{E}(Z, N)+E_{\text {shell }}(Z, N)
$$


For superheavy nuclei $\tilde{E}$ is of the order $-2000 \mathrm{MeV}$ while $E_{\text {shell }}$ fluctuates in the range $-15 \mathrm{MeV} \leq E_{\text {shell }} \leq+15 \mathrm{MeV}$. In MM models the self-consistent coupling of shell structure and bulk properties is replaced by (independent but similar) parameterizations of the the density distribution and the single-particle potentials with $N$ and $Z$. Modern MM models combine a finite-range liquid-drop (YPE) or droplet (FRDM) model for $\tilde{E}$ and a phenomenological single-particle model based on either the Woods-Saxon (WS) or the Folded-Yukawa (FY) potential for the calculation of $E_{\text {shell }}$. The two most widely used models are the FRDM+FY20 and the YPE+WS models21. It is to be noted that loosely-bound systems like superheavy nuclei where the Fermi energy is close to the continuum require a more careful treatment of unbound states thapdone in the standard approach used in large-scale calculations with MM model 22 .

\section{$3 \quad$ Nuclear exotica in superheavy nuclei}

In superheavy nuclei the repulsive Coulomb interaction is not counteracted by the surface tension which leads to a vanishing liquid-drop fission barrier. Therefore the Coulomb field cannot be treated as a small perturbation atop the nuclear mean field, it pushes the protons to the nuclear surface which is counteracted by the symmetry energy restoring a similar distribution of protons and neutrons and the density dependence of the effective interaction restoring the saturation density of nuclear matter (for given asymmetry). With that superheavy nuclei probe the balance of bulk properties of effective interactions. At the same time the large density of single-particle states provides a sensitive probe for even subtle details of the single-particle structure. Again the Coulomb potential induces significant changes in the proton shell structure, compared to lighter nuclei single-particle states with large angular momentum $j$ are lowered compared to small $-j$ states 23 .

Up to now most of the understanding of phenomena in superheavy nuclei was obtained on the basis of MM models. Recent work employing SC models, however, predicts some new phenomena which cannot be consistently described by (current) MM models. Examples are exotic radial density distributions like "semi-bubbles" 24 (which are essential for the appearance of spherical shell closures at $Z=120$ and $N=172$ predicted by some SC models 17 ) and the variation of the surface diffuseness in superheavy nuclei. It is well-known that the appearance of a proton shell at $Z=126$ requires a larger surface diffuseness than assumed ${ }_{i n}$ the standard parameterizations of the FY and WS single-particle potentials25.26. Standard MM models use a parameterization of the radial shape of the density distribution and single-particle potentials that has no free parameters to minimize the binding energy and therefore are not flexible enough to incorporate both of these effects. It can be expected that these models loose their validity for nuclei with large charge number. SC models have to be preferred when describing these phenomena as they make no assumption at all on the profile of the density distributions of protons and neutrons. Besides the limitations of the currently used parameterizations the deeper reason why these phenomena cannot be easily described by MM models is that they are caused by the coupling of single-particle degrees of freedom and bulk properties which becomes more pronounced for loosely bound systems. 


\section{Fits and Parameterizations}

Although they might not contain all physics relevant for superheavy nuclei beyond the known region current MM models give a better description of binding energies than the best SC models26. This is not too surprising as the aims and the fit strategies of MM and SC models are very different: MM models are optimized for the description of masses by adjusting the parameters of the macroscopic part $\tilde{E}$ to all known masses. The density distribution entering the macroscopic part of the model is parameterized as a function of $N$ and $Z$ to reproduce the global systematics of radii, the microscopic potential is adjusted to reproduce systematics of single-particle spectra throughout the chart of nuclei. On the other hand, SC models are designed to give a consistent description of nuclear ground states, singleparticle spectra, collective excitations like giant resonances and rotational bands, and large-amplitude collective motion within the same model. The parameters of the interaction are usually adjusted to masses and data on the charge distribution of selected spherical nuclei, and in some cases to selected data on infinite nuclear matter (INM) to compensate for the small number of sample points in the fit. As the description of single-particle spectra and bulk properties cannot be separated the interactions need not to be adjusted to spectral data with the exception of the spinorbit $(\ell \cdot s)$ interaction. As a purely relativistic effect it is naturally incorporated in the RMF 16 which reproduces data on $\cdot s$ splittings without being adjusted to any data on single-particle spectra at allin. In non-relativistic models - either MM or SC ones - the $\ell \cdot s$ force has to be put in by hand and needs to be adjusted to spectral data.

A detail to be kept in mind when comparing masses from MM and SC models is that in the fit of MM models the absolute error of $\mathcal{E}$ is minimized, while for SC models usually the relative error of $\mathcal{E}$ is minimized 27 which allows for much larger absolute errors in heavy nuclei, up to $5.5 \mathrm{MeV}$ in ${ }^{208} \mathrm{~Pb}$ corresponding to $0.35 \%$.

Besides the technical reason that everything else than a fit to a small sample of spherical nuclei is too time-consuming there are also physics reasons for the usual small sample of fit nuclei. The many-body wave function $\Phi$ of mean-field models breaks symmetries which are obeyed by the effective energy functional and the "exact" wave function. An instructive example for this "symmetry dilemma" is violation of translational symmetry which is unavoidable as the center-of-mass (c.m.) of the nucleus is localized by the mean-field potential. Although $\Phi$ has vanishing total momentum $\langle\mathbf{P}\rangle=0$ it is not an eigenstate of the momentum operator, $\left\langle\mathbf{P}^{2}\right\rangle \neq 0$. This means that $\Phi$ is not the pure ground state but contains an admixture of excited states with finite momentum which decreases the calculated mass. Rigorous restoration of the broken symmetry by means of projection is too costly to be used in large-scale calculations. The method of choice is to estimate the contribution from excited states to the calculated energy and subtract this c.m. correction $E_{\text {c.m. }}$. to obtain the binding energy. An unexpected side-effect is that the nuclear matter properties of effective interactions depend on technical details of the actual c.m. correction performed during the fit as the effective interaction has to compensate for the difference between approximations and the exact value of $E_{\text {c.m. }}$. Often-used simple schemes for c.m. correction lead accidentally to too large 


\begin{tabular}{lccc}
\hline Force & $\begin{array}{c}a_{\text {vol }} \\
{[\mathrm{MeV}]}\end{array}$ & $\begin{array}{c}a_{\text {sym }} \\
{[\mathrm{MeV}]}\end{array}$ & $\begin{array}{c}a_{\text {surf }} \\
{[\mathrm{MeV}]}\end{array}$ \\
\hline SkM $^{*}$ & -15.8 & 30.0 & 17.6 \\
SkP & -15.9 & 30.0 & 18.0 \\
SkI3 & -16.0 & 34.8 & 17.5 \\
SkI4 & -15.9 & 29.5 & 17.3 \\
SLy6 & -15.9 & 32.0 & 17.4 \\
\hline
\end{tabular}

\begin{tabular}{lccc}
\hline Force & $\begin{array}{c}a_{\text {vol }} \\
{[\mathrm{MeV}]}\end{array}$ & $\begin{array}{c}a_{\text {sym }} \\
{[\mathrm{MeV}]}\end{array}$ & $\begin{array}{c}a_{\text {surf }} \\
{[\mathrm{MeV}]}\end{array}$ \\
\hline NL-Z & -16.2 & 41.7 & 17.7 \\
NL-Z2 & -16.1 & 39.0 & \\
NL3 & -16.2 & 37.4 & 18.5 \\
\hline
\end{tabular}

Table 1. Compilation of nuclear matter properties for a number of typical parameter sets. SkM*-SLy6 are Skyrme forces, and NL-Z, NL-Z2, and NL3 RMF forces. $a_{\mathrm{vol}}$ denotes the volume coefficient or energy per nucleon, $a_{\text {sym }}$ the (volume) asymmetry coefficient, and $a_{\text {surf }}$ the surface coefficient. Empirical values for the volume cfficients derived from the liquid-drop model are $a_{\mathrm{vol}}=-16.0 \pm 0.2$ and $a_{\mathrm{sym}}=32.5 \pm 0.5$. See 22 for more details.

a surface coefficient 28 which has visible impact on the extrapolation of the models to heavy systems and large deformation. A similar influence on the results of a fit can be expected for other corrections for spurious motions, namely the rotational and vibrational correction. Luckily their contribution to the binding energycan be suppressed by choosing spherical nuclei with stiff potential energy surfaces27 which explains the usual small sample of fit nuclei.

There are numerous parameterizations of the SHF and RMF models to be found in the literature. Results discussed here are obtained with the SHF interactions SkM*29, SkP30, SLy631, SkI3 and SkI432, and the RMF forces NL333, NL-Z34, and NL-Z217. SkP uses effective mass $m^{*} / m=1$ and is designed to describe both mean-field and pairing interaction. The other Skyrme forces all have smaller effective masses around $m^{*} / m \approx 0.75$. $\mathrm{SkM}^{*}$ was first to deliver acceptable incompressibility and fission properties. SLy6 stems from an attempt to cover properties of pure neutron matter together with normal nuclear ground-state properties, while SkI3 and SkI4 stem from a recent fit including data from exotic nuclei and use a variant of the Skyrme parameterization where the $\ell \cdot s$ force is complemented by an explicit isovector degree-of-freedom 32 They are designed to overcome the different isovector trends of $\ell \cdot s$ coupling between conventional Skyrme forces and the RMF. The RMF parameterizations NL-Z, NL-Z2, and NL3 use the standard non-linear ansatz for the RMF model. NL-Z aims at a best fit to nuclear ground-state properties. NL-Z2 matches exactly the same enlarged set of data like the SkI $x$ forces. NL3 results from a fit including neutron rms radii and nuclear matter data.

\section{Predictive Power}

Table 1 summarizes those nuclear matter properties that can be directly linked to the leading terms of the liquid-drop model

$$
E_{\mathrm{LDM}}=a_{\mathrm{vol}} A+a_{\mathrm{sym}} I^{2} A+a_{\mathrm{surf}} A^{2 / 3}+\ldots
$$

for some typical SHF and RMF forces. Most non-relativistic (relativistic) interactions agree among each other in the values for $a_{\mathrm{vol}}$ and $a_{\mathrm{sym}}$, but relativistic and 


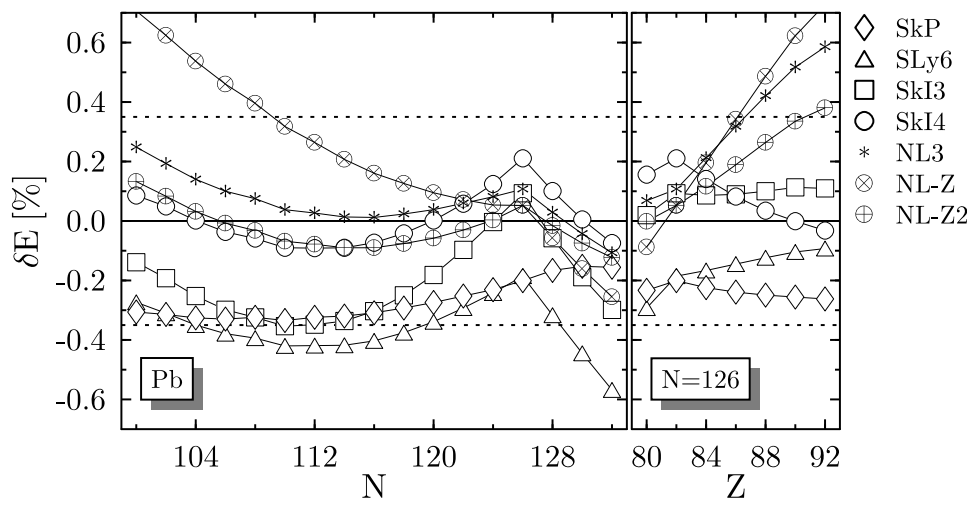

Figure 1. Relative error $\delta E=\left(E_{\text {calc }}-E_{\text {expt }}\right) / E_{\text {expt }}$ (in \%) of the binding energy for the chain of $\mathrm{Pb}$ isotopes and the chain of $N=126$ isotones. Negative values denote under-bound, positive values over-bound nuclei. 7 horizontal lines at $\pm 0.35 \%$ indicate the relative error in binding energy allowed for good fits 1632.

non-relativistic interactions differ significantly. While the Skyrme force values are close to the empirical ones, the RMF forces give larger $a_{\text {vol }}$ and $a_{\text {sym. }}$. work much better in that respect. It is important to note, Not all values for Skyrme interactions are predictions, for the SLy6, SkP, and $\mathrm{SkM}^{*}$ forces nuclear matter properties were used as input data during their fit. As already mentioned above the predicted value for $a_{\text {surf }}$ is accidentally correlated to the scheme for c.m. correction used in the fit of the interaction 28 , forces with simple c.m. correction like SkP and NL3 have systematically larger $a_{\text {surf }}$ than the others.

A first impression of the quality of mean-field models for finite nuclei is provided by the systematics of the relative error of binding energies, see Figs. 1 and 2 for typical results. For the chain of spherical $\mathrm{Pb}$ isotopes the results remain essentially within the bounds of $0.35 \%$ allowed in the fits, while the spherical $N=126$ isotones reveal already some unresolved trends in the RMF interactions. The slope of $\delta E$ corresponds to an error of the two-nucleon separation energies. Unlike modern SHF interactions which have four or even five independent isovector terms, the RMF has one isovector term only which seems to be insufficient. The small change in the isovector coupling between $\mathrm{NL}-\mathrm{Z}$ and $\mathrm{NL}-\mathrm{Z} 2$ improves visibly the trends with $N$ but at the same time worsens the trends with $Z$. But one has to be careful as both $I$ and $A$ change along isotopic and isotonic chains which mixes trends in the isovector and isoscalar channel. These can be separated plotting $\delta E$ against $I$ and $A$, see Fig. 2 with data for superheavy nuclei. It can be expected that in this extrapolation of the models the $\delta E$ spread more than in the case of the lighter nuclei. It is gratifying, however, to see that most of the interactions stay within or at least close to the bounds of $0.35 \%$ error. When plotted versus $A$ one obtains essentially flat curves for all SHF forces, while there is a small but visible slope for the RMF forces that points at an unresolved isoscalar trend. When plotted versus $I$ all forces (perhaps with the exception of $\mathrm{SkP}$ ) show slopes which point at unresolved trends in the isovector channel, even for modern forces like NL3 or 


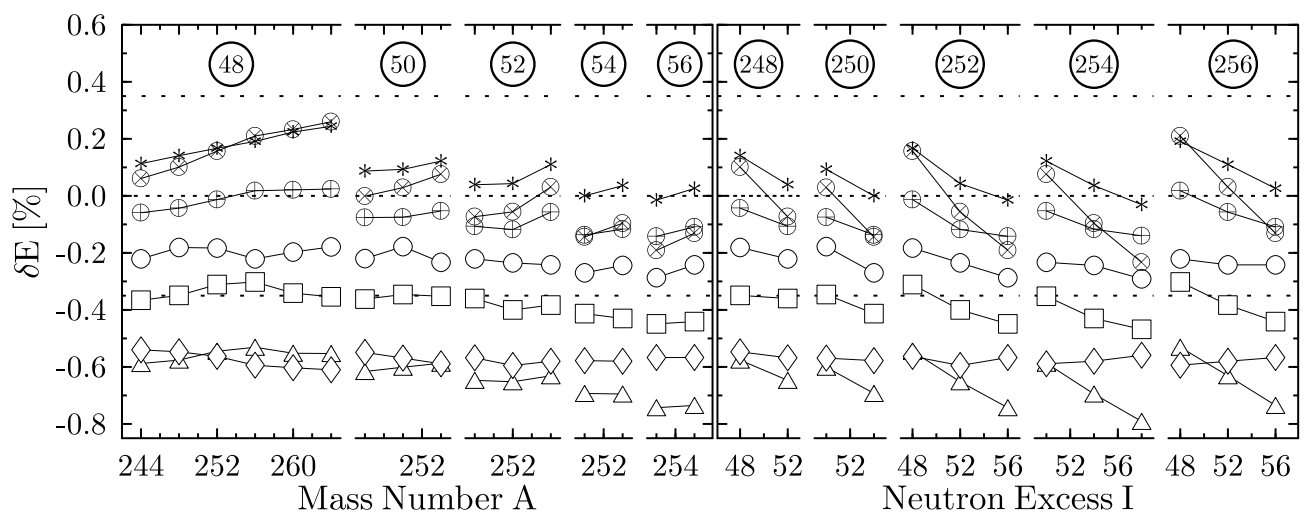

Figure 2. Relative error the binding energy for the heaviest deformed nuclei where the mass is known plotted for constant $I=N-Z$ against $A=N+Z$ (left panel) and vice versa (right panel) to separate trends in the isoscatq $g$ nd isovector channels of the effective interactions. Symbols as in Fig. 1. Data are taken from 3536

SLy6 fitted with bias on good isovector properties. One has to be careful with the interpretation of Fig. 2 since the range of known masses in $I$ is rather small and wrong trends in the "macroscopic part" of the SC models might interfere with local flaws in the microscopic part. An example for that is the peak in the $\delta E$ around ${ }^{208} \mathrm{~Pb}$ in the left panel of Fig. 1 1 which hints at an unresolved shell effect. It is to be noted that missing shell effects might also correspond to correlations beyond the mean-field level, see the example of c.m. correlations discussed in 28 .

As there are no experimental data on spectral properties of superheavy nuclei, the predictive power of the models for single-particle spectra has to be examined looking at lighter nuclei, see Fig. 3. None of the existing parameterizations of $\mathrm{SC}$ or MM models is able to give a proper description of the level ordering and the spin-orbit splitting in heavy nuclei such as ${ }^{132} \mathrm{Sn}$ or ${ }^{208} \mathrm{~Pb}$. Levels with large angular momentum are usually pushed up too far in the single-particle spectrum and all non-relativistic models (either SC and MM ones) show a wrong trend of the spin-orbit splitting with $A$ and therefore usually overestimate the proton spin-orbit splitting in heavy nuclei. This is devastating for interactions where the spin-orbit coupling constant is adjusted to data for ${ }^{16} \mathrm{O}$. Including data on heavy nuclei in the fit gives better overall agreement but cannot remove the overall wrong trend as can be seen from the example of SkP. It is surprising that Skyrme forces with an additional degree of freedom in the spin-orbit interaction (SkI3, SkI4) perform worse in that respect than standard forces (SkP, SLy6). The overall performance of the RMF interactions is much better.

As most mean-field models predict the same level-ordering in the superheavy region, already slight changes in the relative distances among the interactions lead to different magic numbers, see Fig. 1 for typical results. Large spin-orbit splitting as in case of the FY and SkI4 models favors $Z=114$, but as these interactions overestimate the spin-orbit splitting of proton states in heavy nuclei this prediction is very doubtful. Non-relativistic models in general prefer $N=184$ for neutrons, 

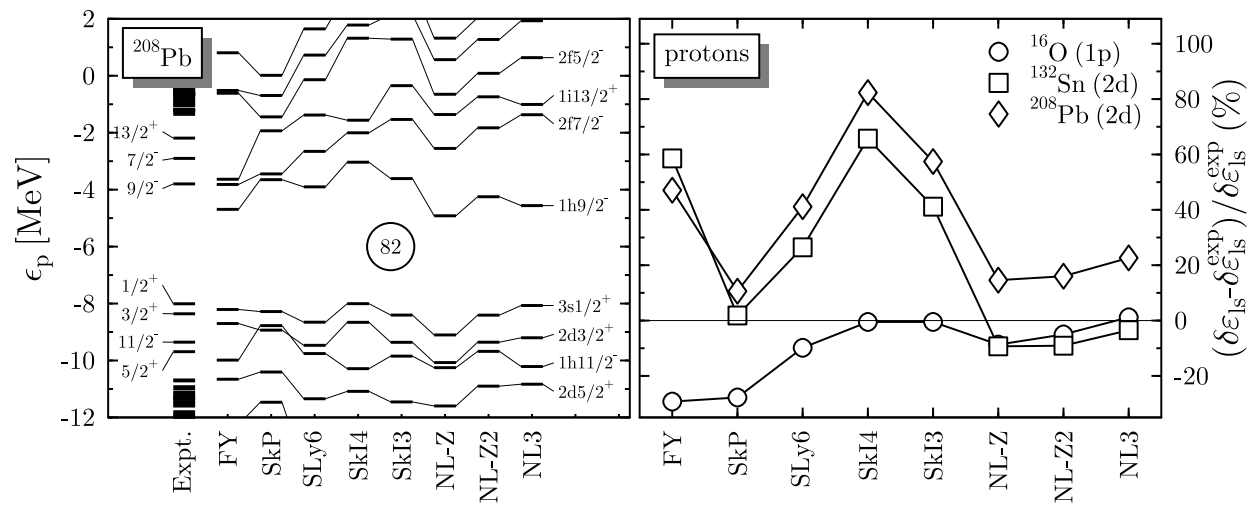

Figure 3. Single-particle spectra of the protons in ${ }^{208} \mathrm{~Pb}$ (left panel) and relative error of the spin-orbit splittings $\delta \epsilon_{\mathrm{ls}}=\left(\epsilon_{\mathrm{ls}, \text { calc }}-\epsilon_{\mathrm{ls} \text {,expt }}\right) / \epsilon_{\mathrm{ls} \text {,expt }}$ (in \%) of proton states close to the Fermi surface in ${ }^{16} \mathrm{O},{ }^{132} \mathrm{Sn}$ and ${ }^{208} \mathrm{~Pb}$ for the interactions as indicated. Negative errors denote calculated values which are too small. FY dengte the folded-Yukawa single-particle potential widely used in MM models. Data are taken from 17 .

while small spin-orbit splitting in connection with "semi-bubble" shapes leads to $Z=120$ and $N=172$ as it happens for SkI3 and the relativistic forces NL3, NL-Z, and NL-Z2. SC interactions with large effective mass close to $m^{*} / m \approx 1.0$ like SkP give an average level density so large that most of the shell effects are washed out and the proton shell is shifted to $Z=126$. A common feature of spherical shells as predicted by SC models is their strong nucleon-number dependence 7 37: While the spectra for the non-SC FY model remain basically unchanged, the spectra from SC models change dramatically going from ${ }_{184}^{298} 114$ to ${ }_{172}^{292} 120$, the spherical shells at
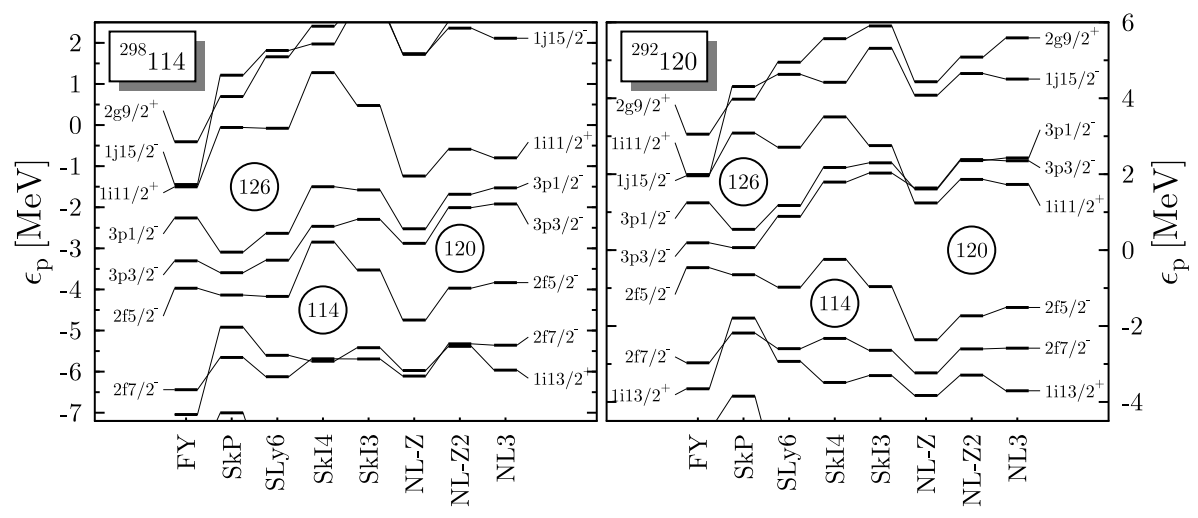

Figure 4. Single-particle spectra of the protons in ${ }_{184}^{298} 114$ and ${ }_{172}^{292} 120$ at spherical shape for the interactions as indicated. Note that in spite of the is actually deformed for most Skyrme interactions 38 , 39, while the strong $N=184$ shell stabilizes for most interactions the spherical shape of ${ }_{184}^{298} 114$. Data are taken from 17 . 


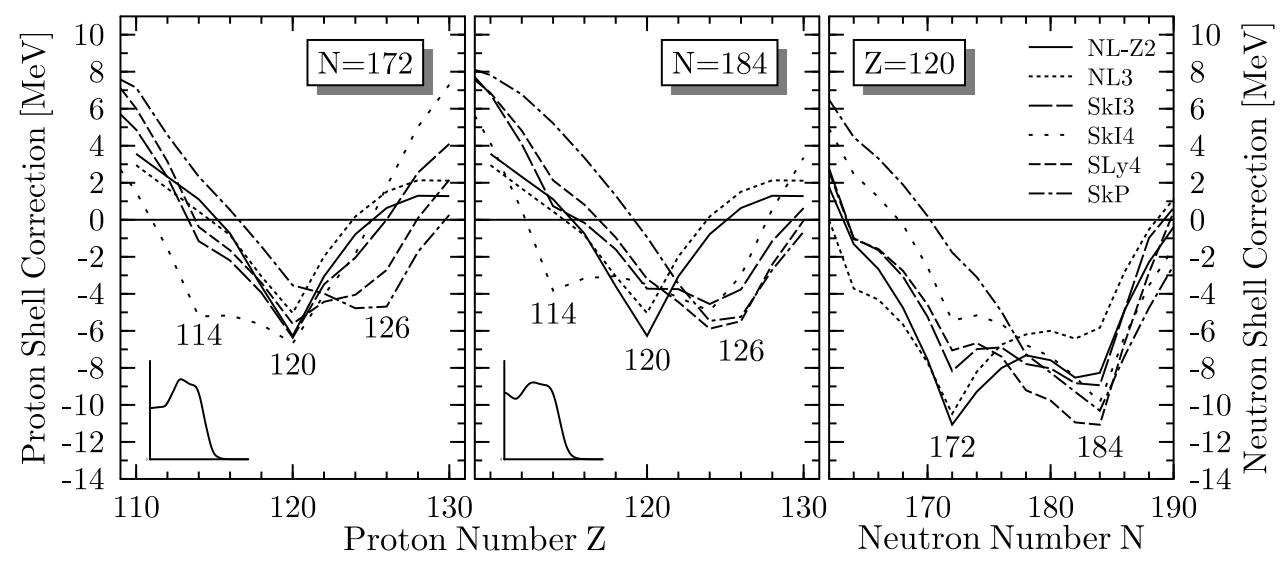

Figure 5. Proton shell correction extracted from calculated self-consistent binding energies of the $N=172$ and $N=184$ isotones (left and middle panel) and neutron shell correction for the $Z=120$ isotopes. The same energy scale is used for all panels. Note that for the majority of Skyrme interactions all $N=172$ isotones are predicted to be deformed. The inserts show the density profile for ${ }^{292} 120$ (left panel) and ${ }^{304} 120$ (middle panel) calculated with NL-Z2. They demonstrate the strong neutron-number dependence of the "semi-bubble" shapes which are responsible for the magic numbers $Z=120$ and $N=172$. Data are taken from 22 .

$Z=120, Z=126$ and $N=172$ are all restricted to a narrow range of $N$ and $Z$, a magic $N=184$ often excludes a shell closure at $Z=120$.

The appearance of gaps in the single-particle spectra alone is not sufficient to stabilize a superheavy nucleus. Much more important is the extra binding and its strong shape dependence obtained from a smaller than average level density. The shell correction can be viewed as the natural measure for this "shell effect" and provides a powerful tool to analyze also results obtained in fully self-consistent calculations, see Fig. 5. It has to be emphasized that the shell correction is not equivalent to the gap in the single-particle spectrum and that a quantitative comparison of the two quantities cannot be made. For $N=172$ most of the Skyrme forces give a magic $Z=120$, while for $N=184$ the minimum is shifted to $Z=124$ 126 in all cases. RMF forces give consistently different results, the strongest shell effect is at $Z=120$ independent on the neutron number. Most interactions predict also a broad region of negative neutron shell correction. Again a systematic difference between the models: while the RMF points at $N=172$ as the dominant neutron shell, all SHF forces predict $N=184$. Note that shell corrections for neutrons are much larger than for protons. This partly explains the finding that spherical ground states of superheayn nuclei are usually correlated with magic neutron numbers, not proton numbers23.36.40. All this suggests that the "island of stability" is not so much coupled to particular shell closures but a region of nuclei with low level density which is also found in MM model 20 . Fig. 5 demonstrates also that it is nearly impossible to discriminate between models giving different shell closures by looking at systematics of binding energies or $Q_{\alpha}$ values alone. Although the proton shell correction might be peaked at different points, its variation 


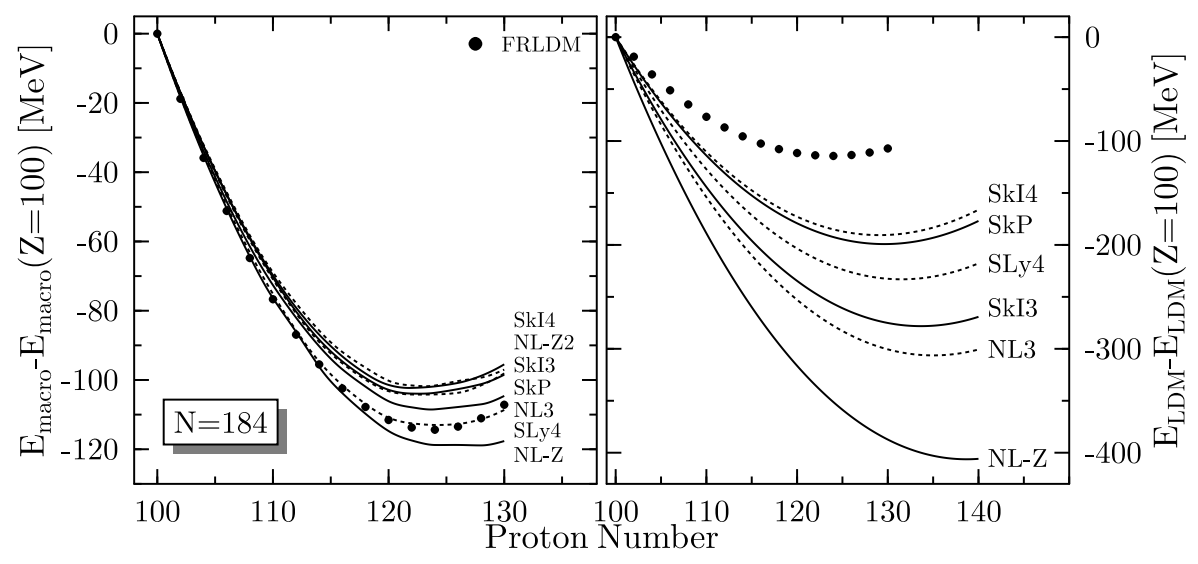

Figure 6. Macroscopic energy $\tilde{E}$ extracted from calculated self-consistent binding energies (left panel) and macroscopic energy $E_{\mathrm{LDM}}$ from the LDM expansion (5) of the binding energy (right panel) for the chain of $N=184$ isotones. The phenomenological macroscopic energy from the YPE model is given for comparison. Note that the scales of the two panels are very different. To illustrate the $Z$ dependence all energies are normalized to the YPE value at $Z=100$. Data taken from Ref.22

is often too small to be visible within the uncertainty of SC models.

The shell correction does not only extract the "shell effect" from the selfconsistent binding energies, at the same time one obtains the macroscopic part $\tilde{E}$ of the binding energy. A typical example is given in the left panel of Fig. 5 . Owing to its optimization as a mass formula and its fit which includes also superheavy nuclei the YPE model can be expected to give a very good description of the macroscopic energy in this region and therefore can serve as reference. The curves for the various interactions show considerable splitting which reflects of course the findings from Figs. 1 and 2. This also confirms again the finding discussed above that the predictive power of an effective interaction for binding energy systematics is fairly independent of its predictive power for shell effects. On one hand SLy4 and NL3 give very similar values for $\tilde{E}$ as the YPE model (but remember the small errors in their trends found in Fig. 2) although they predict different magic numbers, on the other hand those forces that give the best description of binding energies independently on their disagreement on the next shell closure (SkI3, SkI4, SkP, NL-Z2) give all very similar $\tilde{E}$ but differ now significantly from the YPE model.

The right panel of Fig. 6 shows the macroscopic energy $E_{\mathrm{LDM}}$ from the LDM expansion of the binding energy (5) using the values given in Table 1. Surprisingly $\tilde{E}$ and $E_{\mathrm{LDM}}$ differ on the order of $100 \mathrm{MeV}$. Only for $E_{\mathrm{LDM}}$ the ordering of the interactions is according to their value for $a_{\mathrm{sym}}$ as one naively expects. This demonstrates that the leptodermous expansion with nuclear matter parameters does not work even for superheavy nuclei, finite-size effects are still important which also means that the use of nuclear matter parameters as pseudo-observables in the fit of SC models might be dangerous.

Most of the recent new data on superheavy nuclei are $Q_{\alpha}$ values. Their sys- 


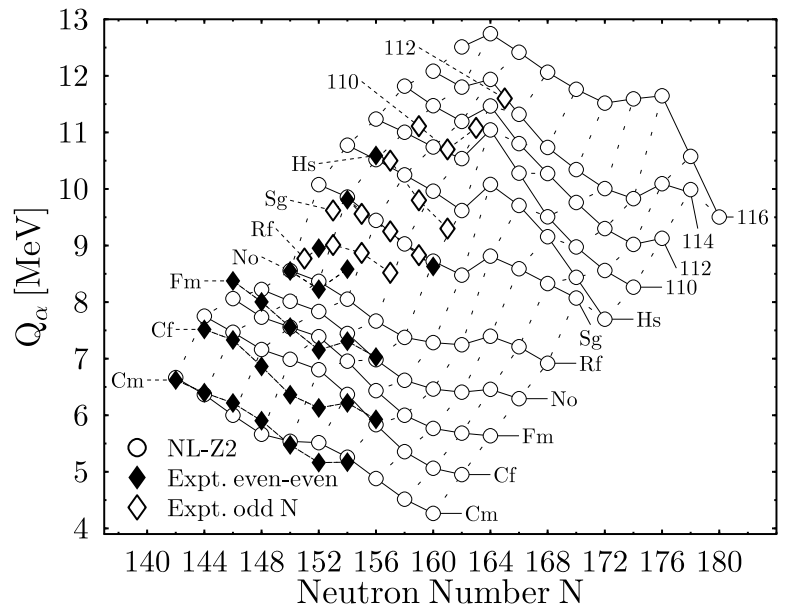

Figure 7. $Q_{\alpha}$ values of even-even nuclei calculated with NL-Z2 (open circles) compared with experimental values for even-even nuclei (filled diamonds) and odd- $N$ isotopes (open diamonds) of the heaviest even $Z$ elements. The data for odd $-N$ nuclei have to be handled carefully, some of these might correspond to transitions involving excited states, and due to blocking effects of the ground-state-to-ground-state values might differ on the order of $500 \mathrm{keV}$ from the systematics of $Q \alpha$ for even-even nuclei. Including data for nuclei with $Z>116$ leads to overlapping curves, therefore those are omitted in the plot. Data taken from 10

tematics reflect all properties discussed above. As $\alpha$-decay chains have $I=$ const. the isoscalar channel of $\tilde{E}$ mainly determines the overall slope of the $Q_{\alpha}$, while the isovector channel of $\tilde{E}$ mainly shifts the whole curves around. Shell effects bend the curves locally, leading to kinks and peaks. A model has to give a perfect description of all these properties to reproduce experimental data throughout the superheavy region. The quality of NL-Z2 for the $Q_{\alpha}$ is shown in Fig. 7. The overall description of the data is very good with the exception of some nuclei around $Z=104$ where it overestimates a deformed shell closure while the deformed $N=152$ shell is shifted to $N=150$. The latter is a problem from which virtually all SC models suffere 36 .

Fig. 8 compares some very recent data with calculations. In view of the uncertainties, the SC SLy4 and NL-Z2 give a very good description of the data for the decay chain of ${ }_{165}^{27112}$ (NL-Z2 agrees for $Z>104$ only as can be expected from Fig. (7) and reproduces the $N=162$ shell effect which cannot be seen in the FRDM+FY predictions. While all models give similar predictions for this wellestablished chain, the spread among the models is much larger for the new chains. All models with the exception of YPE+WS show spherical or deformed shells which cannot be seen in the data.

Comparing predictions with the recent data for the even-even ${ }_{176}^{292} 116$ decay chain (which still have to be viewed as preliminary), see the right panel of Fig. 8 , it is most interesting that the data agree with calculated values from interactions which give different predictions for the spherical magic numbers, i.e. SkI4 ( $Z=114$, $N=184), \operatorname{SLy} 6(Z=126, N=184)$, and NL3 $(Z=120, N=172)$. All other interactions show wrong overall trends of the $Q_{\alpha}$ or pronounced deformed shells 

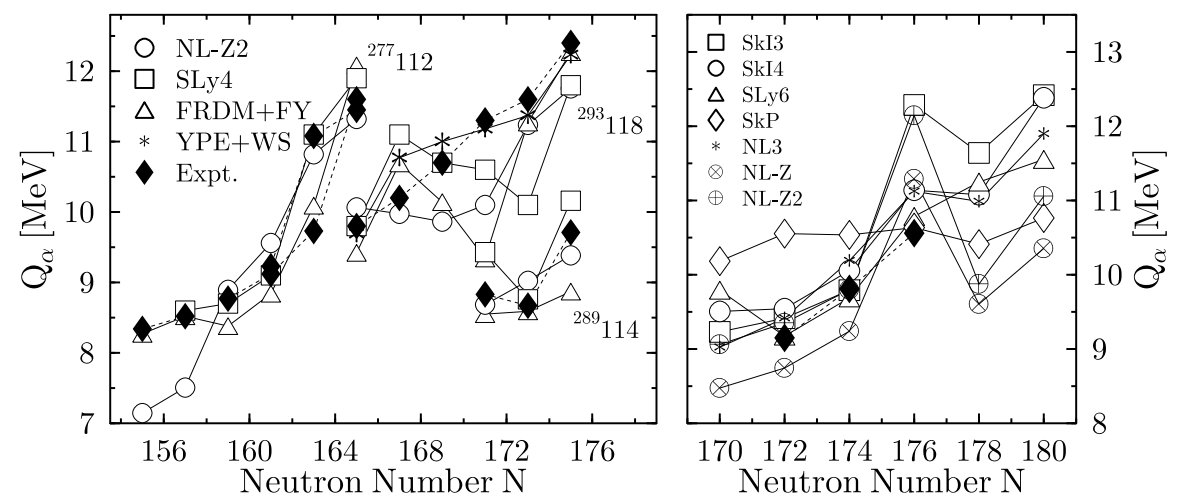

Figure 8. Left panel: Comparison of experimental and calculated $Q_{\alpha}$ values for the decay chains of ${ }_{165}^{27} 112,{ }_{175}^{289} 114$, and ${ }_{175}^{293} 118$, in the latter two cases following the mass and charge assignment of the experimental groups. In the ${ }_{165}^{277} 112$ chain two distinct branches leading through different states of the intermediate nuclei are known. The calculated values from NL-Z2 and SLy 4 connect the lowest states with positive parity in all cases (in the new chains only ${ }_{175}^{289} 114$ and ${ }_{167}^{277} 110$ are predicted by SLy4 and NL-Z2 to have ground states with negative parity), while the FRDM+FY and YPE+WS data are ground state to ground state values. Right panel: $Q_{\alpha}$ values of nuclei in the decay-chain ${ }_{180}^{300} 120 \rightarrow{ }_{178}^{296} 118 \rightarrow \ldots{ }_{168}^{276} \mathrm{Hs}$ as predicted by the mean-field models as indicated compared with preliminary experimental data.

in disagreement with the data or even both. The large difference between NL-Z and NL-Z2 is caused by their difference in symmetry energy although both forces predict the same shell structure. Once again all this demonstrates that predictions for spherical shell closures and binding energy systematics are fairly independent.

One of the reasons for the poor description of the new data is that -independent on the actual location of the shell closures - these nuclei are located in a region of transitional nuclei with very soft potential energy surfaces which amplify small differences in the shell structure. At the same time this adds a large uncertainty to the predictions as correlations beyond the mean-field level give a non-negligible contribution to binding energy differences which washes out shell effects visible in the results from mean-field calculation迎.

\section{Conclusions}

Combining the findings for average trends of binding energies and single-particle spectra it has to be said that none of the current models is able to describe all available data. Errors in the macroscopic part of the models have to be distinguished from errors in the shell structure. A good description of binding energy trends is not neccesarily an indicator for the predictive power concerning shell effects. Looking at "macroscopic" observables, a single parameterization of a model is not representative for the model while observables sensitive to shell effects reveal systematic differences between SHF and RMF. The good overall description of spin-orbit splittings by the RMF gives some preferrence for its predictions for magic numbers, but the undoubtedly missing isovector degrees of freedom in the 
RMF once included might also feed back to its predictions for superheavy shell closures. More research in that direction has to be done.

\section{Acknowledgments}

I would like to express my gratitute to all my collaborators with whom the ideas and results presented here were develloped over the years, in particular T. Bürvenich, S. Ćwiok, P.-H. Heenen, A. Kruppa, J. A. Maruhn, W. Nazarewicz, P.-G. Reinhard, K. Rutz, T. Schilling, T. Vertse, and W. Greiner. I am grateful for many fruitful discussions on experimental data and their interpretation with S. Hofmann, G. Münzenberg, V. Ninov, and Yu. Ts. Oganessian, and I want to thank the organizers of the International Conference on "Fusion dynamics at the extremes" for

the kind invitation to present these results. A travel grant from the Herrmann Willkomm-Stiftung is gratefully acknowledged.

\section{References}

1. S. Hofmann, Rep. Prog. Phys. 61, 639 (1998).

2. S. Hofmann, G. Münzenberg, Rev. Mod. Phys. 72, 733 (2000).

3. H. Meldner, Proc. of the Int. Symposium on "Why and how should we investigate nuclides far off the stability line", Lysekil, Sweden, August 21-27, 1966, W. Forsling, C. J. Herrlander, H. Ryde [edts.], Ark. Fys. 36, 593 (1967).

4. H. Meldner, Phys. Rev. 178, 1815 (1969).

5. S. G. Nilsson, C. F. Tsang, A. Sobiczewski, Z. Szymanski, S. Wycech, C. Gustafson, I.-L. Lamm, P. Möller, B. Nilsson, Nucl. Phys. A131, 1 (1969).

6. Yu. Ts. Oganessian et al., Eur. Phys. J. A5, 68 (1999), Phys. Rev. Lett. 83, 3154 (1999), Nature 400, 209 (1999), Phys. Rev. C 62, 041604(R) (2000), and private communication (2000).

7. V. Ninov et al., Phys. Rev. Lett. 83, 1104 (1999).

8. R. Smolańczuk, Phys. Rev. C 59, 2634 (1999).

9. S. Ćwiok, W. Nazarewicz, P.-H. Heenen, Phys. Rev. Lett. 83, 1108 (1999).

10. M. Bender, Phys. Rev. C 61, 031302(R) (2000).

11. P. Armbruster, Eur. Phys. J. A7, 23 (2000).

12. P. Hohenberg, W. Kohn, Phys. Rev. B 136, 864 (1964).

13. W. Kohn, L. J. Sham, Phys. Rev. A140, 1133 (1965).

14. I. Zh. Petkov, M. V. Stoitsov, Nuclear Density Functional Theory, Clarendon Press, Oxford, 1991.

15. J. Dobaczewski, J. Dudek, Proc. of "High Angular Momentum Phenomena, Workshop in honour of Zdzisław Szymański", Piaski, Poland, August 23-26, 1995, Acta Physica Polonica B27, 45 (1996).

16. P.-G. Reinhard, Rep. Prog. Phys. 52, 439 (1989).

17. M. Bender, K. Rutz, P.-G. Reinhard, J. A. Maruhn, W. Greiner, Phys. Rev. C 60, 034304 (1999).

18. V. M. Strutinsky, Nucl. Phys. A95, 420 (1967), ibid. A122, 1 (1968).

19. S. G. Nilsson, I. Ragnarsson, Shapes and Shells in Nuclear Structure, Cambridge University Press, 1995. 
20. P. Möller, J. R. Nix, Nucl. Phys. A536, 84 (1992), J. Phys. G20, 1681 (1994).

21. R. Smolańczuk, Phys. Rev. C 56, 812 (1997).

22. A. T. Kruppa, M. Bender, W. Nazarewicz, P.-G. Reinhard, T. Vertse, S. Ćwiok, Phys. Rev. C 61, 034313 (2000)

23. S. Ćwiok, J. Dobaczewski, P.-H. Heenen, P. Magierski, W. Nazarewicz, Nucl. Phys. A611, 211 (1996).

24. J. Dechargé, J.-F. Berger, K. Dietrich, M. S. Weiss, Phys. Lett. B451, 275 (1999), K. Dietrich, contribution to these proceedings.

25. W. D. Myers, W. J. Swiatecki, Phys. Rev. C 58, 3368 (1998).

26. W. D. Myers, W. J. Swiatecki, Phys. Rev. C 60, 054313 (1999).

27. J. Friedrich, P.-G. Reinhard, Phys. Rev. C 33, 335 (1986).

28. M. Bender, K. Rutz, P.-G. Reinhard, J. A. Maruhn, Eur. Phys. J. A8, 59 (2000).

29. J. Bartel, P. Quentin, M. Brack, C. Guet, H.-B. Håkansson, Nucl. Phys. A386, 79 (1982).

30. J. Dobaczewski, H. Flocard, J. Treiner, Nucl. Phys. A422, 103 (1984).

31. E. Chabanat, P. Bonche, P. Haensel, J. Meyer, R. Schaeffer, Nucl. Phys. A635, 231 (1998), A643, 441 (1998).

32. P.-G. Reinhard, H. Flocard, Nucl. Phys. A584, 467 (1995).

33. G. A. Lalazissis, J. König, P. Ring, Phys. Rev. C 55, 540 (1997).

34. M. Rufa, P.-G. Reinhard, J. A. Maruhn, W. Greiner, M. R. Strayer, Phys. Rev. C 38, 390 (1989).

35. M. Bender, K. Rutz, T. Bürvenich, P.-G. Reinhard, J. A. Maruhn, W. Greiner, Proc. of "Nuclear Shapes and Motions - A symposium in honor of Ray Nix", Santa Fe, New Mexico, October 25-27, 1998 APH N.S. Heavy Ion Physics 10, 185 (1999).

36. T. Bürvenich, K. Rutz, M. Bender, P.-G. Reinhard, J. A. Maruhn, W. Greiner, Eur. Phys. J. A3, 139 (1998).

37. K. Rutz, M. Bender, T. Bürvenich, T. Schilling, P.-G. Reinhard, J. A. Maruhn, W. Greiner, Phys. Rev. C56, 238 (1997).

38. M. Bender, K, Rutz, P.-G. Reinhard, J. A. Maruhn, W. Greiner, Phys. Rev. C 58, 2126 (1998).

39. S. Ćwiok, M. Bender, P.-H. Heenen, W. Nazarewicz, unpublished results.

40. G. A. Lalazissis, M. M. Sharma, P. Ring, Y. K. Gambhir, Nucl. Phys. A608, $202(1996)$.

41. P.-G. Reinhard, M. Bender, T. Bürvenich, T. Cornelius, P. Fleischer, J. A. Maruhn, Proc. of the "Tours Symposium on Nuclear Physics IV", Tours, France, September 2000, in press. 